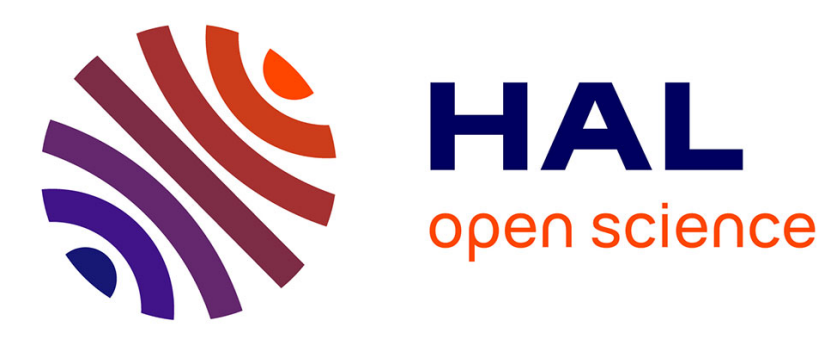

\title{
Action publique territoriale
}

Alain Faure

\section{To cite this version:}

Alain Faure. Action publique territoriale. Alistair Cole, Sébastien Guigner, Romain Pasquier. Dictionnaire des politiques territoriales, Presses de Sciences Po, pp.25-33, 2011. halshs-00660363

\section{HAL Id: halshs-00660363 \\ https://shs.hal.science/halshs-00660363}

Submitted on 16 Jan 2012

HAL is a multi-disciplinary open access archive for the deposit and dissemination of scientific research documents, whether they are published or not. The documents may come from teaching and research institutions in France or abroad, or from public or private research centers.
L'archive ouverte pluridisciplinaire HAL, est destinée au dépôt et à la diffusion de documents scientifiques de niveau recherche, publiés ou non, émanant des établissements d'enseignement et de recherche français ou étrangers, des laboratoires publics ou privés. 


\title{
Action publique territoriale
}

\author{
Alain Faure (CNRS IEP Grenoble)
}

Pourquoi et comment la formule "action publique territoriale " s'est-elle invitée ces dernières années dans les cercles savants et dans les milieux experts pour qualifier les enjeux de gestion des collectivités locales, mais aussi pour désigner certaines programmes d'action initiés par les Etats et même certaines priorités imposées par l'Union européenne [fonds structurels] ? La " territorialité " de l'action collective évoque-t-elle une rupture de sens par rapport aux notions d'action publique locale, de politiques publiques locales, d'administration locale [administration locale], de pouvoir local, de gouvernement local, de développement local ?... Pour ouvrir cette discussion, sans doute faut-il s'interroger dans une perspective foucaldienne sur ce qui relie "les mots et les choses", sur le changement des discours au cours du temps, sur les régularités discursives des couches de savoirs qui délimitent ce qu'une époque peut ou non penser. A cet égard, il semble que l'évolution sémantique du local vers le territorial offre un cas intéressant de réenchantement de l'action publique sur les leitmotivs de l'efficacité et de la proximité [proximité]: d'une part la formule semble signifier un mouvement de professionnalisation des interventions publiques directement impulsé par des collectivités locales, d'autre part elle suggère que ces mesures et programmes possèdent un supplément d'âme dès lors que les individus sont au plus près de la décision publique. II apparaît ainsi que le qualificatif " territorial » véhicule une représentation de l'action publique à la fois performative et vertueuse: c'est la promesse d'une action collective avec plus de responsabilité pour les grandes collectivités locales, une meilleure capacité d'expertise des citoyens sur les décisions publiques et même une vigilance accrue face aux dérives bureaucratiques ou idéologiques. Le terme suggère une conception morale du vivre ensemble où se précisent des croyances précises sur le bien commun.

Notons au préalable qu'il est difficile d'évoquer la portée narrative de cette formule sans opérer un bref retour sur l'histoire du terme "territoire » dans les différentes littératures scientifiques à l'échelon international. Deux clivages principaux en marquent la genèse. Le premier concerne l'extrême diversité des appréhensions académiques au sein des sciences sociales, avec des définitions non compatibles entre ethnologues, géographes, juristes, économistes ou politistes. Le second concerne l'usage du terme par culture nationale, que ce soit la lecture républicaine singulière adoptée en France depuis la Révolution française (Alliès 1980), la variété des traditions sur le local government en Europe [gouvernement local] ou encore la diversité des conceptualisations de la spatialité politique dans les cinq continents [espace]. Ce double clivage a longtemps brouillé les tentatives pour imaginer des passerelles entre le territoire géographique des espaces vécus, le territoire politique des institutions, le territoire naturel des espaces protégés, le territoire ritualisé des communautés, le territoire militaire des relations internationales... De cette genèse impossible, retenons simplement l'extraordinaire prégnance des sentiers intellectuels tant disciplinaires que nationaux et l'absence de vision commune et de savoirs cumulables.

Malgré cela, une convergence langagière semble se dessiner depuis quelques années, notamment en Europe, sur la territorialité de l'action publique. Le virage est apparemment engagé sous la pression conjuguée de trois évolutions: le ciblage de certaines politiques européennes sur des espaces infranationaux en difficulté ; le développement de métiers et de savoir-faire professionnels spécifiquement appliqués aux enjeux de développement local [développement local] et de développement durable [développement durable] ; les réformes institutionnelles qui transfèrent des compétences considérables aux échelons des régions et des villes (Lefèvre 2009). C'est vraisemblablement cette triple dynamique de rationalisation et de professionnalisation des interventions publiques qui constitue le premier indice de territorialisation de l'action publique et l'apparition, dans le langage des professionnels, de la catégorie 
" action publique territoriale ". L'évolution se formalise avec la structuration de filières spécialisées et de nouveaux métiers dans les champs de l'aménagement, de l'environnement ou de l'action sociale; elle s'alimente du recrutement d'agents plus qualifiés dans les collectivités locales et de l'invention de formes spécifiques d'expertises localisées; elle s'organise avec la création de cursus universitaires et de formations techniques; elle se concrétise avec la mise en place de dispositifs publics ciblés sur le rural en déclin, l'urbain en crise, les zones de reconversion industrielle, les zones littorales, les centre-ville, les régions périurbaines, les territoires de montagne (Faure Négrier 2007)... L'évolution s'est récemment cristallisée sur des mots valise comme le " projet de territoire " [projet] et la "gouvernance territoriale " (Pasquier Simoulin Weisbein 2007) [gouvernance territoriale], notions qui véhiculent l'idée que l'efficacité des programmes dépend de formes renouvelées de coordination, de contractualisation et de régulation collectives à l'échelon local [contrat]. En France, cet engouement est à la fois analysé et encouragé par de nombreux travaux en sciences sociales appliquées (urbanisme, management, environnement, sciences économiques, développement durable...). Des analystes font le constat que les territoires deviennent des acteurs du changement parce qu'ils interviennent en tant qu'opérateurs, vecteurs ou encore opportunités mobilisant des ressources, des énergies et des potentialités de toute sorte (Vanier 2009).

Cette perception d'une modernité par la performance territoriale nous entraine sur un second registre discursif émergent, celui de la citoyenneté par la proximité. Nombreux sont les cercles savants et experts qui diffusent des diagnostics insistant sur le fait que les collectivités locales sont les mieux placées pour prendre le pouls des citoyens en matière de programmation et de gestion des services publics. En quelques années, l'argument de la proximité s'est imposé comme un sésame de la légitimité des politiques publiques, et ce souvent au détriment des arènes traditionnelles de médiation investies par les instances représentatives et les corps intermédiaires (syndicats, partis, chambres consulaires, associations, corporations) [proximité]. Pour bien gouverner, il faudrait donc se situer au plus près des individus, en prise directe avec leurs attentes et leurs besoins. Les élus placés loin du terrain ou sans ancrage " territorial " seraient même disqualifiés pour poser les " bons " diagnostics. On retrouve ce message à la fois dans de nombreuses arènes militantes et au cœur de toutes les réformes engagées depuis les années 80 par les Etats nationaux en matière de décentralisation et de déconcentration [décentralisation]. La volonté de transférer aux collectivités locales des compétences conséquentes repose d'abord sur l'argument que la focale des administrations locales permet un traitement plus "juste " de la demande sociale. On constate à cet égard que pratiquement tous les niveaux de collectivité locale expérimentent dorénavant des dispositifs locaux de participation et de concertation, sur la conviction qu'une "bonne " politique publique passe par une appréhension directe et sans intermédiaire des problèmes individuels à résoudre [démocratie participative].

En termes de science politique, cette double évolution nous incite à souligner les nouveaux éléments de croyances que l'action publique territoriale génère dans le champ du politique et des pratiques démocratiques par rapport aux modèles canoniques de l'Etat providence et de la légitimité par les gouvernements représentatifs. Dans la lignée des travaux pionniers d'E. Evans-Pritchard, l'ethnologue Marc Abélès avait étudié dans les années 80 comment les scènes politiques communales et départementales généraient sur le cas français leurs propres critères d'éligibilité territoriale pour sélectionner les élus locaux (Abélès 1989). L'auteur avait identifié les attributs sociaux et culturels permettant aux candidats d'incarner une évocation précise du territoire tout en l'insérant dans l'univers des réseaux de pouvoir professionnels et étatiques balisés par des événements fondateurs et des marquages cérémoniels. Cette façon de décrypter le territoire comme "producteur et manipulateur de territorialité " prend une importance particulière à l'heure où les élites politiques locales acquièrent des responsabilités considérables dans le pilotage des politiques publiques [leadership ; élites territoriales]. La potentielle territorialité du politique semble en effet une piste particulièrement stimulante pour décrypter le sens des récits locaux sur le bien commun [intérêt local]. Si l'on opère un bref retour sur les hypothèses de l'Ecole de Chicago concernant l'esprit des lieux et l'analyse des phénomènes de socialisation, il faut convenir que le lien qui s'établit entre les mondes sociaux locaux et les identités collectives conditionne pour partie les enjeux de domination et de représentation politiques [identité]. Cette perspective située et historicisée permet alors de questionner les fondations démocratiques de la vie dans la cité. Dans le présent dictionnaire, la piste est suggérée sur trois facettes : la façon dont les gouvernements nationaux tentent de contrôler le double processus contemporain de territorialisation et d'institutionnalisation de 
l'action collective [Etat local], les mécanismes inédits de subsidiarité qui placent différents niveaux d'intervention publique en concurrence et en tension [échelle d'action publique], l'enchevêtrement des ordres politiques générés sur différents espaces géographiques institutionnalisés (la représentation politique, les politiques publiques, les appartenances) [territoire]. Tous ces débats académiques participent d'une réflexion sur le différentiel démocratique qui caractérise l'action publique dite territoriale, avec au moins trois types de variables en interaction : les traumatismes historiques qui imprègnent le fonctionnement de chaque territoire ; les promesses du politique qui structurent la relation de confiance (et de défiance) entre les élus locaux et les électeurs [clientélisme] ; les croyances sur l'intérêt général qui sont spécifiquement produites dans chaque système politique local pour légitimer les priorités d'action publique [système politique local ; intérêt local]. Cet ensemble de représentations en interactions nous informe sur le complexe processus de différenciation à l'œuvre, la construction sociale et politique de l'action publique territoriale relevant de motifs qui peuvent être perçus et exprimés de façon très différente selon chaque contexte spatial et temporel. L'analyse de cette démocratie différentielle en formation permet ainsi d'orienter la réflexion sur les liens qui se tissent entre les faits sociaux et les croyances politiques. Sans doute la démarche implique-t-elle d'adopter une grille des savoirs particulièrement sensible à la façon dont les pratiques sont pensées et délimitées dans chaque ordre local. Sur les plans théorique et méthodologique, l'entrée suggère un défi de taille: convoquer des outils d'analyse sur les formes d'énonciation du politique qui dépendent de savoirs disciplinaires disparates et souvent hyperspécialisés (micro-histoire, anthropologie, linguistique, études électorales, sociologie politique, approche cognitive des politiques publiques, philosophie, psychologie sociale...).

\section{5 références bibliographiques}

Abélès (Marc), Jours tranquilles en 89. Ethnologie politique d'un département français, Paris, Odile Jacob, 366 p. , 1989

Alliès (Paul), L'invention du territoire, Grenoble, Presses Universitaires de Grenoble, 180 p. , 1980

Faure (Alain), Négrier (Emmanuel) dir., Les politiques publiques à l'épreuve de l'action locale. Critiques de la décentralisation, L'Harmattan, 2007, 240 p.

Lefèvre (Christian), Gouverner les métropoles, Paris, L.G.D.J., coll. Politiques locales, 115 p. , 2009

Pasquier (Romain), Simoulin (Vincent), Weisbein (Julien), La gouvernance territoriale. Pratiques, discours et théories, LGGJ Droit et Société, Série Politique, 235 p. , 2007

Vanier (Martin) (dir.), Territoires, territorialité, territorialisation, Controverses et perspectives, Rennes, PUR, coll. "Espace et territoires», 228 p., 2009 